\title{
Single-Port Repair of Diastasis Recti and Umbilical Hernia Combined with Abdominoplasty
}

\author{
Hanh Tran, FRACS, Isidro Turingan, RN, Mai Tran, Marta Zajkowska, RN, Vincent Lam, FRACS, \\ Wayne Hawthorne, MD
}

Discipline of Surgery, Sydney Medical School, The University of Sydney, Westmead Hospital, Westmead, Australia (Drs. H. Tran, Lam, Hawthorne).

Sydney Hernia Specialists Clinic, Sydney, Australia (Turingan, M. Tran, Zajkowska).

\begin{abstract}
Introduction: Evidence-based treatment of abdominal hernias involves the use of prosthetic mesh. However, the most commonly used method of treatment of diastasis of the recti involves plication with non-absorbable sutures as part of an abdominoplasty procedure. This case report describes single-port laparoscopic repair of diastasis of recti and umbilical hernia with prosthetic mesh after plication with slowly absorbable sutures combined with abdominoplasty.

Technique Description: Our patient is a 36-year-old woman with severe diastasis of the recti, umbilical hernia and an excessive amount of redundant skin after two previous pregnancies and caesarean sections. After raising the upper abdominal flap, a single-port was placed in the left upper quadrant and the ligamenturn teres was divided. The diastasis of the recti and umbilical hernia were plicated under direct vision with continuous and interrupted slowly absorbable sutures before an antiadhesive mesh was placed behind the repair with $6 \mathrm{~cm}$ overlap, transfixed in 4 quadrants and tacked in place with non-absorbable tacks in a double-crown technique. The left upper quadrant wound was closed with slowly absorbable sutures. The excess skin was removed and fibrin sealant was sprayed in the subcutaneous space to minimize the risk of serorna formation without using drains.
\end{abstract}

Discussion: Combining single-port laparoscopic repair of diastasis of recti and umbilical hemia repair minimizes inadvertent suturing of abdominal contents during plication, the risks of port site hernias associated with conventional multipart repair and permanently reinforced the midline weakness while achieving "scarless" surgery.

Key Words: Single incision laparoscopic surgery, Diastasis of recti Abdominoplasty.

Citation Tran H, Turingan I, Tran M, Zajkowska M, Lam V, Hawthorne W. Single-port repair of diastasis recti and umbilical hernia combined with abdominoplasty. CRSLS e2014.00213. DOI: 10.4293/CRSLS.2014.00213

Copyright (C) 2014 SLS This is an open-access article distributed under the terms of the Creative Commons Attribution-Noncommercial-ShareAlike 3.0 Unported license, which permits unrestricted noncommercial use, distribution, and reproduction in any medium, provided the original author and source are credited.

The authors thank Dr. Lionel Chang, FRACS for his assistance in performing the abdominoplasty.

Address correspondence to: Hanh Tran, FRACS, Level 2, 195 Macquarie Street, Sydney, NSW 2000, Australia. Tel: +61 2 9221 1043 , Fax: +61 2 8076 3229, E-mail: info@sydneyherniaspecialists.com.au

\section{INTRODUCTION}

The surgical treatment of diastasis of the recti remains controversial because it is anatomically not a hernia and, hence, it does not have dangerous sequelae such as bowel obstruction and perforation. Yet, for patients who are relatively young and are affected by the condition, it has adverse physical implications (because they cannot exercise without feeling pain or discomfort) and psychological implications because of the severe amount of excess wrinkled abdominal skin, making it extremely unsightly. The conventional treatment is abdominoplasty with or without plication of the recti. ${ }^{1-3}$ However, there are 2 problems with this approach. First, the plication is performed "blindly," and given the fact that there is very little separation of the thinned rectus sheath and the underlying intestine/stomach, though there is coverage by omentum, it is not possible to be completely certain that inadvertent suturing of the bowel wall does not take place. This can have both immediate and longer-term implications in terms of adhesions and their sequelae. Second, the currently accepted best method for treatment of any hernia 
defect is prosthetic reinforcement with wide overlap of $\geq 5 \mathrm{~cm}$ in all dimensions. ${ }^{4,5}$ However, this is not routine practice by most plastic surgeons with regard to the repair of diastasis of the recti.6,7 Even if the surgeon places an onlay mesh, it will have the same significant complications, in particular infection, which is known to occur in up to $53 \%$ of all open ventral hernia repairs. ${ }^{8}$

Pure laparoscopic repair of diastasis of the recti can easily be performed, but it does not address the problem of the overlying excess skin, which is the main complaint in such patients. 9 The laparoscopic ventral hernia repair has continued to evolve from a concept of tension-free repair to the realization that defects, if their diameter is small enough (ie, $<6 \mathrm{~cm}$ ), should now be closed because this not only improves abdominal wall functioning but also allows wider overlap of the "defects." The technique of defect closure both in an extracorporeal manner and in an intraperitoneal manner is well described, ${ }^{10}$ and theoretically, this can be used to repair diastasis of the recti.

Our unit began performing single-incision laparoscopic surgery (SILS) in October 2009, and we reported the first 100 cases of single-incision laparoscopic totally extraperitoneal inguinal hernia repair in June 2010. ${ }^{11}$ To date, we have performed $>600$ cases of single-incision laparoscopic inguinal hernia repair and $>80$ ventral (including parastomal) hernia repairs with excellent results. ${ }^{11-13} \mathrm{We}$ have also completed a prospective randomized controlled study (NCT01660048) comparing single and multiport total extraperitoneal inguinal herniorrhaphy.

Despite numerous reports showing the superiority of laparoscopic repair for ventral hernias, including low infection rates, low recurrence rates, and early return to work and/or physical activities, its adoption rate has been estimated to be $\leq 10 \%$ in most developed countries. The reasons for its low adoption rate, as compared with laparoscopic inguinal hernia repair, include the fact that, traditionally, the laparoscopic repair does not close the defect and this can result in persistent unsightly seroma formation. The practice of defect closure has become increasingly more widely practiced, and it is known to reduce seroma formation in addition to improving abdominal wall functioning. ${ }^{10}$

To our knowledge, we report the first case of singleincision laparoscopic repair of diastasis of the recti and umbilical hernia combined with abdominoplasty. This article aims to illustrate the important principles of SILS, defect closure under direct vision, prosthetic mesh reinforcement of the defect, and an attempt to reduce intraperitoneal adhesions and postoperative seroma formation with fibrin sealant while achieving the desired result with excision of unsightly skin excess. This study was approved by the Independent Review Board of Holroyd Private Hospital.

\section{CASE REPORT}

The patient was a 36-year-old woman, with a body mass index (BMI) of $22.5 \mathrm{~kg} / \mathrm{m}^{2}$, who had 2 previous uncomplicated cesarean sections. She noticed an umbilical hernia during the second pregnancy. After delivery, she also noticed a large amount of redundant abdominal skin, in addition to an increasing abdominal bulge, which was most prominent at the level of the umbilicus (Figure 1). She reported that she regularly worked out at a gym to keep fit and to eliminate excess skin but that she had to wear an abdominal binder for comfort during exercise. She sought advice from the senior author and was informed that an abdominoplasty to remove her unsightly skin excess, combined with a modified single-incision laparoscopic repair of the umbilical hernia and diastasis of the recti with plication, under direct vision for safety reasons, as well as intraperitoneal mesh reinforcement, would offer the best repair and cosmetic results. Like many of our patients, she had performed extensive research online and was aware of the current literature on the subject. She consented to undergo the proposed procedure.

After induction of anesthesia and a single dose of cefazolin, $1 \mathrm{~g}$, intravenously, the patient underwent catheteriza-

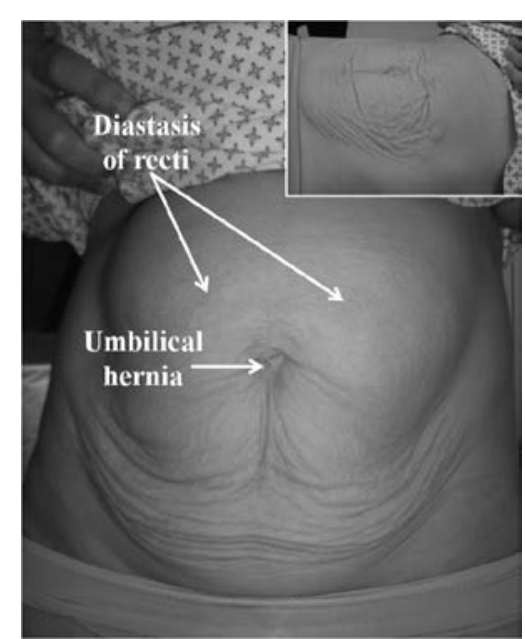

Figure 1. Preoperative photograph showing significant bulging of the central and upper abdomen as a result of diastasis of the recti with concomitant umbilical hernia. The inset shows unsightly excess abdominal skin. 
tion. The patient was prepared from the epigastrium to the mid thighs and draped with iodine-impregnated adhesive cover (Ioban; 3M, St Paul, Minnesota). The plastic surgeon then raised the flap of skin and freed it sufficiently for excision of the excess skin and closure of the wound without tension (Figure 2). The herniologist performed the repair of the diastasis of the recti and umbilical hernia as follows: The medial edges of the recti were marked with a permanent marker. After infiltration of the left upper quadrant with $20 \mathrm{~mL}$ of $0.5 \%$ Bupivaccaine with 1:200000 epinephrine, the muscle layers were split in the line of their fibers before the peritoneum was entered for placement of an SILS port. Three 5-mm blunt trocars were inserted, with the anterior trocar being used for insertion of a $52-\mathrm{cm} / 5.5-\mathrm{mm} / 30^{\circ}$ angle laparoscope. Modified dissection techniques, namely "inline" and "chopsticks," were used to overcome the relative loss of triangulation associated with SILS. The ligamentum teres was then taken down superiorly to the xiphisternum (Figure 3). The peritoneum overlying the pubic symphysis was also incised transversely and reflected inferiorly. The anterior $5-\mathrm{mm}$ trocar was then replaced by a $10-\mathrm{mm}$ trocar within the SILS port for insertion of a $10-\mathrm{mm} / 30^{\circ}$ angle laparoscope. The insufflating pressure was reduced to $8 \mathrm{~mm} \mathrm{Hg}$, and by use of a large curved No. 0 PDS suture (Ethicon, Somerville, New Jersey), the stretched rectus sheath was plicated under direct vision such that every entry of the needle was clearly visualized with the laparoscope. A continuous suture was then placed from the epigastrium to the pubic symphysis before being pulled at both ends to approximate the recti. Additional interrupted No. 0 PDS sutures were placed to reinforce the repair, again under direct vision (Figure 3). Slowly dissolving nonpermanent monofilament sutures were used for the plication of the

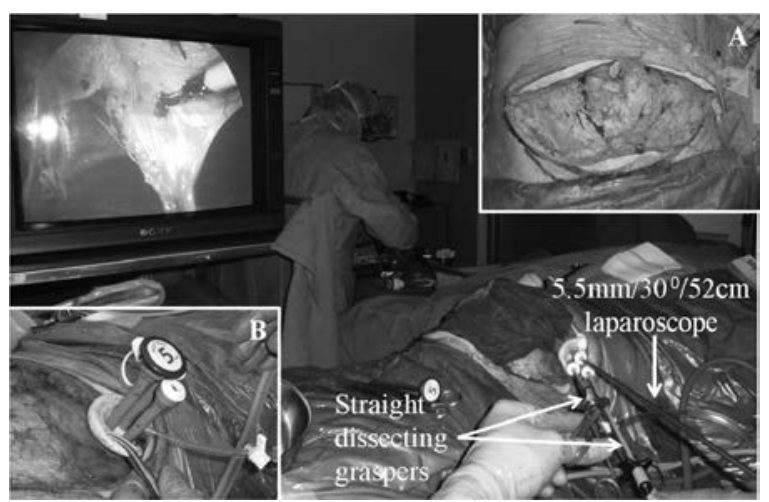

Figure 2. Insertion of SILS port under subcutaneous flap in left upper quadrant with dissecting instruments and laparoscope. A, Excision of excess abdominal skin with medial edges of recti marked. B, Close-up view of SILS port with three 5-mm trocars. recti for 2 reasons: First, multiple knots were present under the thin subcutaneous flap, and these would become palpable in the long-term if they were made of permanent suture, such as nylon. Second, there is no need for permanent sutures because the prosthetic mesh repair maintains the permanency of the plication. It was also noted during the plication of the recti that there was a gas leak at the suture sites, even before the divarication was approximated under low insufflating pressures, and that the abdominal wall musculature, especially laterally below the level of the umbilicus, was exceptionally thin. Therefore, placement of 5-mm trocars, as would occur with conventional multiport laparoscopic surgery, would further increase the air leak, causing difficulties with maintaining the trocars in position and increasing the risk of port-site hernias. These problems were negated by SILS.

Measurements were taken from the epigastrium to the pubic symphysis with the insufflating pressure being reduced to $4 \mathrm{~mm} \mathrm{Hg}$. The mesh dimension was determined to be $12 \mathrm{~cm}$ (transverse) and $22 \mathrm{~cm}$ (longitudinally). The mesh was rolled up, like a scroll, longitudinally so that it could be inserted through the $10-\mathrm{mm}$ trocar under direct vision, with one of the other $5-\mathrm{mm}$ trocars being used to house the 5-mm laparoscope. Transfascial No. 0 PDS sutures were then placed superiorly and laterally before the mesh was fixed with nonabsorbable staples (Protack; Covidien, Norwalk, Connecticut) in a double-crown technique. Fibrin sealant (Tisseel Duo; Baxter, Vienna, Austria) $(2 \mathrm{~mL})$ was then sprayed along the periphery of the mesh and the staples for the purpose of adhesion prevention. After deflation, the left upper quadrant wound was closed in layers with No. 0 PDS sutures. The abdominoplasty was then recommenced in a standard fashion with the excess skin being removed, and a hole was made in the upper flap to reconstruct the umbilicus. Before wound closure, $4 \mathrm{~mL}$ of fibrin sealant was sprayed evenly into the subcutaneous space, and no drain was used (Figure 4). The patient was asked to wear an abdominal binder for 6 weeks and was followed up at 1 week, 6 weeks, and 6 months, with plans to see her annually for 5 years.

The patient was in the hospital for 2 days mainly because she had 2 very young children at home and would have felt pressured to do work, potentially putting stress on the wound. Her postoperative course was uneventful; she was able to return to her office work within 2 weeks. She was exceedingly happy with the result and has remained the same 6 months after surgery (Figure 5). 
Single-Port Repair of Diastasis Recti and Umbilical Hernia Combined with Abdominoplasty, Tran $\mathrm{H}$ et al.
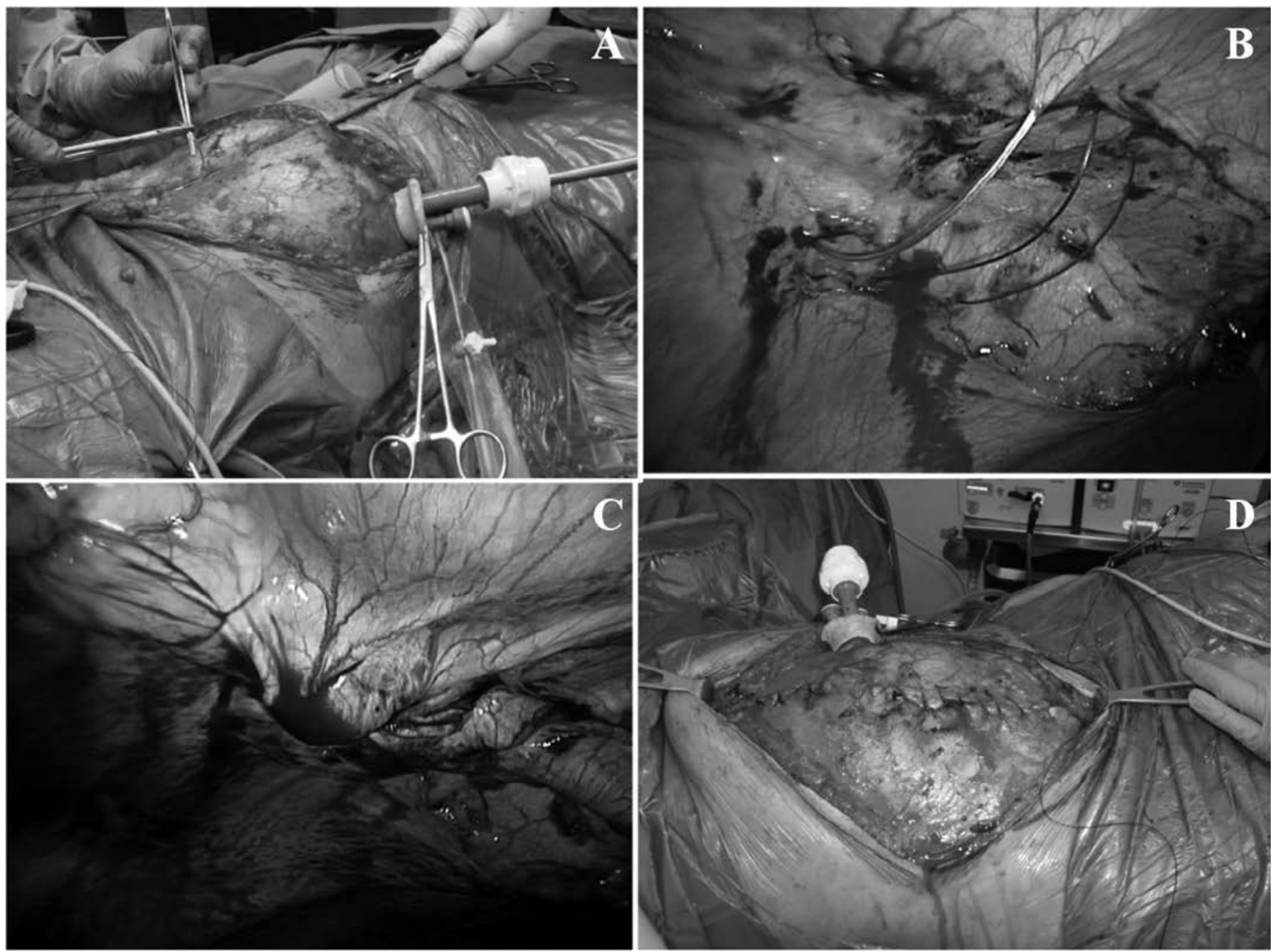

Figure 3. A, External view of approximation of recti. B, Laparoscopic visualization with passing of each suture. C, Recti being approximated. D, Placement of further interrupted sutures to reinforce approximation of recti.

\section{DISCUSSION}

The treatment of diastasis of the recti remains controversial even though abdominoplasty has been the treatment of choice to correct excess skin and fat for $>1$ century. Grazer $^{1}$ was one of the first authors to describe the socalled bikini-line incision, and although he was able to reduce anterior projection of the abdominal wall by aponeurotic suturing in the midline, the procedure did little to reduce the diameter of the waist. This possibly illustrates the fact that suture plication of the midline may not result in permanent repair as was hoped. Bozola and Psillakis ${ }^{14}$ first suggested suture plication of the external oblique musculature after raising it in a belt-like manner and were able to reduce the diameter of the waist. This implied an understanding that muscles, not aponeurosis, give rise to the strength of the repair.

Tadiparthi et $\mathrm{al}^{7}$ reported a series of 28 consecutive patients over a 5-year period who underwent rectus plication during abdominoplasty with No. 0 looped nylon. These patients had a BMI of $26 \mathrm{~kg} / \mathrm{m}^{2}$, and $82 \%$ had previous abdominal surgery including cesarean section. Using ultrasonography to assess the degree of rectus separation, Tadiparthi et al concluded that there was a significant reduction in the mean distance between the rectus muscles before surgery and 12 months postoperatively. They asserted that there were no suture-related complications. This latter assertion, of course, would not include any long-term sequelae from potential inadvertent stitch- 

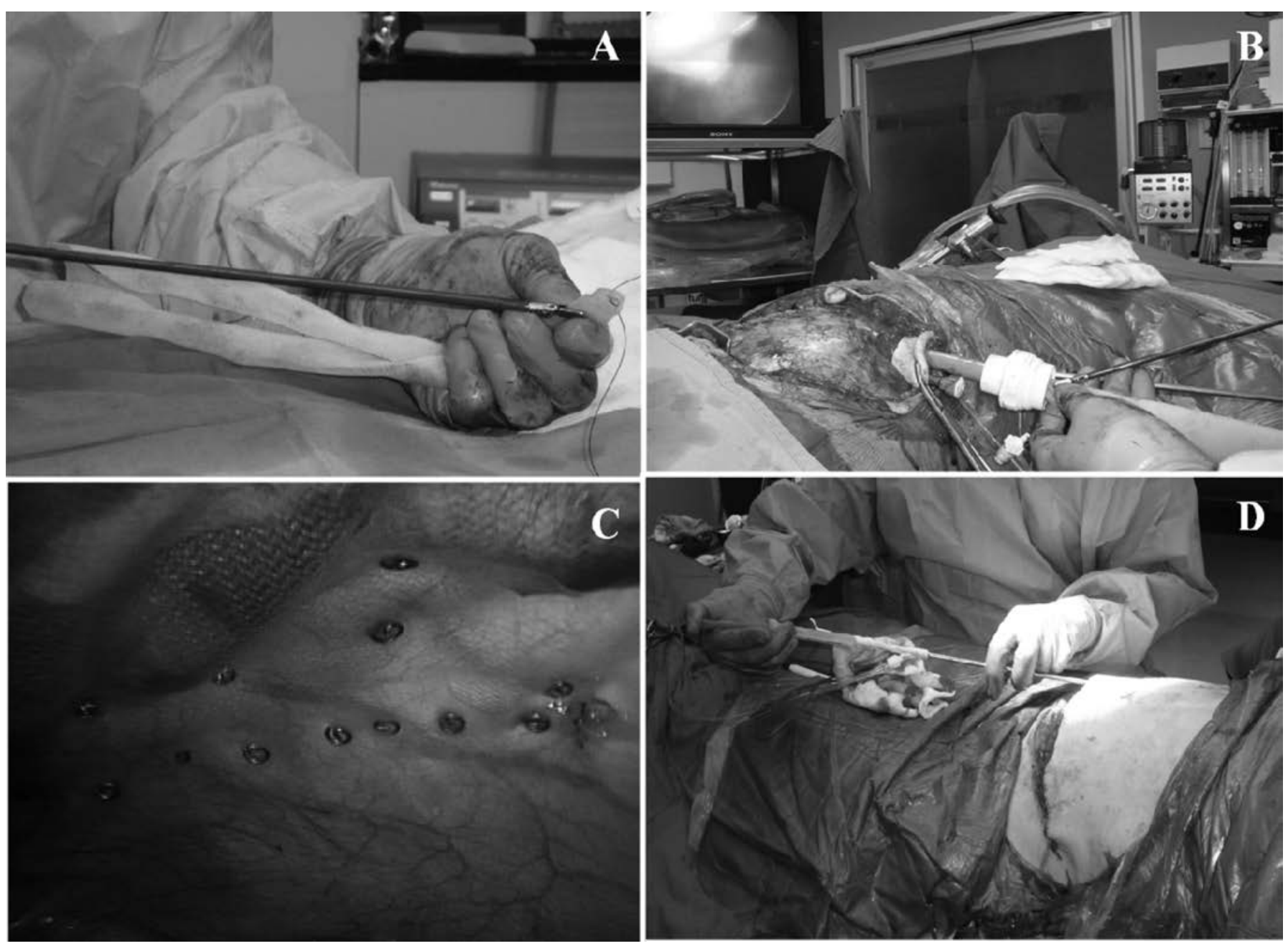

Figure 4. A, Grasping of folded mesh with "dolphin" graspers. B, Insertion into peritoneal cavity through 10-mm port. C, Placement of mesh intraperitoneally with double-crown layer of staples. D, Spraying of fibrin sealant under skin flap.

ing of the bowel during plication. Palanivelu et al ${ }^{9}$ described a method of laparoscopic plication of the recti with the "Venetian blinds" technique combined with mesh reinforcement for patients with diastasis of the recti. The study included 18 patients with a mean BMI of $28.6 \mathrm{~kg} / \mathrm{m}^{2}$. There was no recurrence after a follow-up period of 6 to 48 months, and the authors concluded that the use of prosthetic mesh repair was mandatory to prevent recurrence.

This divergence of views illustrates the difference in appreciation of the underlying pathology. Whereas plastic surgeons accept that there is weakness and stretching of the rectus sheath, it is generally accepted by them that plication alone is sufficient. Conversely, hernia surgeons, as exemplified by Palanivelu et al, ${ }^{9}$ would not contem- plate repairing any kind of abdominal wall defect without prosthetic mesh placement. This assertion is borne out by prospective randomized controlled studies comparing suture versus prosthetic mesh repair for hernias, confirming categorically that the latter offers a far superior repair. However, the pure laparoscopic repair does not address the main reason why these patients seek help-principally to eliminate the large amount of redundant skin such that an abdominoplasty is mandatory.

Our patient represents a typical patient demographic profile in that she had had 2 previous cesarean sections, had an average BMI, and had a large amount of redundant skin that was impossible to eliminate because of the diastasis of the recti, which was most pronounced at the level of the umbilicus. Using the most advanced single- 

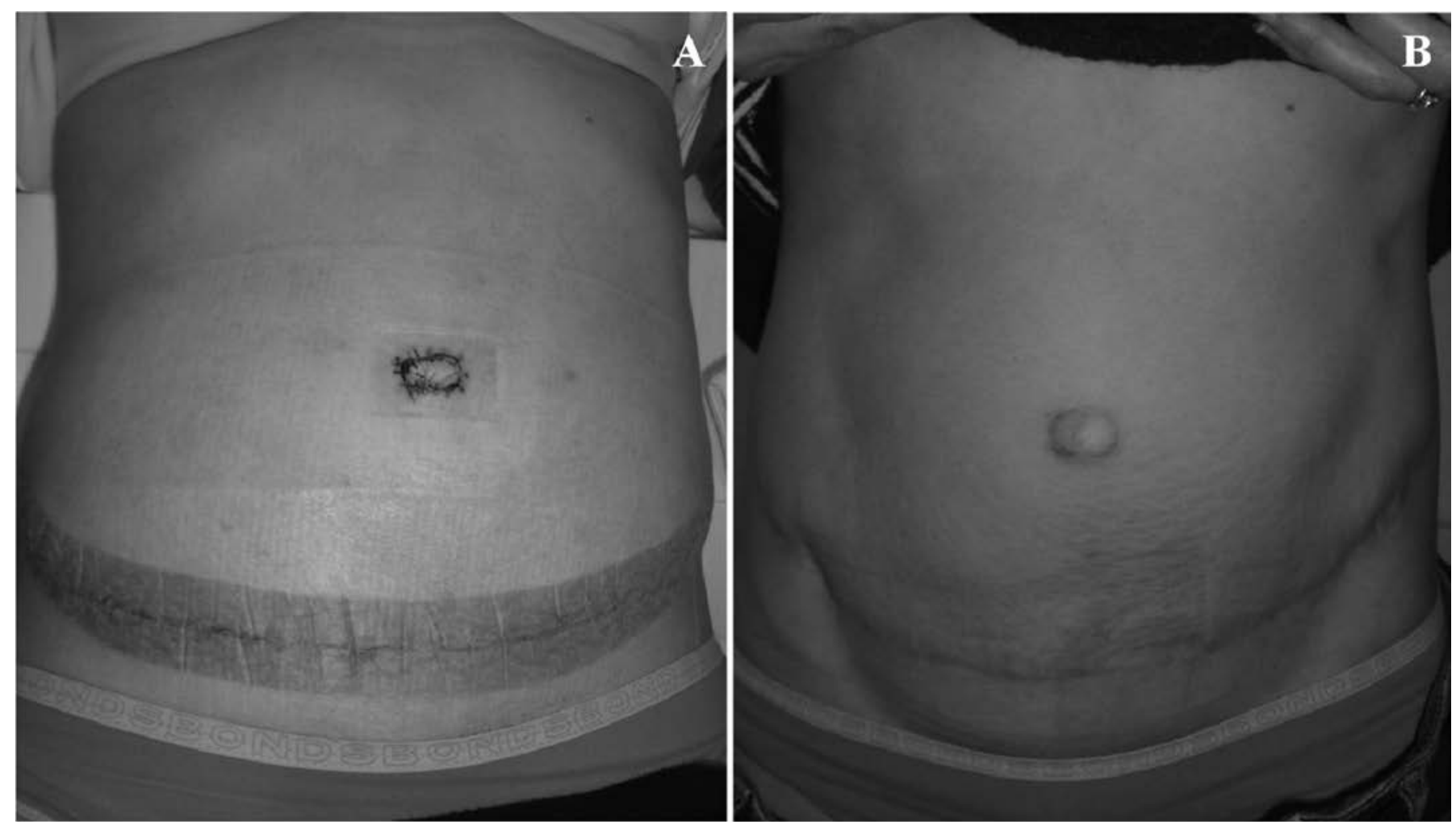

Figure 5. No evidence of seroma or hematoma formation was present 10 days postoperatively (A), and the patient had a flattened abdomen with no evidence of excess skin or recurrence of diastasis of the recti 6 months postoperatively (B).

incision laparoscopic technique minimized parietal trauma and decreased the potential for port-site hernia formation. The latter aspect is particularly important because it was noted that the abdominal wall musculature below the umbilicus was "paper thin," and if conventional multiport laparoscopy had been performed, 2 additional $5-\mathrm{mm}$ ports would have had to be placed into this area. In addition to increasing the difficulties with air leakage around the ports and maintaining the ports in position, this would have significantly increased the risk of port-site hernias. SILS allowed plication of the recti under direct vision and repair of the umbilical hernia, and it reinforced the abdominal wall in the midline with prosthetic mesh. Additional refinements of this technique include the use of absorbable sutures for plication to minimize the risk of persistent suture materials acting as a nidus for infection, as well as being persistent and palpable foreign material in thin patients, and the use of fibrin sealant both to reduce intra-abdominal adhesions and to reduce the risk of seroma formation. ${ }^{15}$ The latter also negated the use of drains that, apart from being cumbersome, can also act as a source of infection.
In the quest for scarless surgery and abolition or reduction of parietal trauma, SILS probably represents one of the most important innovations in minimally invasive surgery in the past 2 decades. Given the fact that it has only really been in existence for 5 years, thousands of cases have been performed across many surgical disciplines ${ }^{16}$, including general surgery (cholecystectomy, ${ }^{17}$ hernia repairs, ${ }^{18}$ appendectomy, ${ }^{19}$ and colorectal resections ${ }^{20}$ ), urology, ${ }^{21}$ and gynecology. ${ }^{22}$ Collaboration between specialties, in this case herniology and plastic surgery, enables an old problem, namely diastasis of the recti, to be addressed in a different light, although further work in terms of patient numbers and longer-term follow-up will determine whether this new concept should be universally adopted.

\section{CONCLUSION}

Combined abdominoplasty and plication of the recti under direct vision with single-incision laparoscopic mesh reinforcement for the treatment of diastasis of the recti resulted in a safe and highly successful repair. Routine adoption of SILS and collaborative work between laparo- 
scopic herniologists and plastic surgeons will provide improved patient care.

\section{References:}

1. Grazer FM. Abdominoplasty. Plast Reconstr Surg. 1973; 51(6):617-623.

2. Pollock H, Pollock T. Progressive tension sutures: a technique to reduce local complications in abdominoplasty. Plast Reconstr Surg. 2000;105(7):2583-2586, discussion 25872588.

3. Angio LG, Piazzese E, Pacile V, et al. The surgical treatment of the diastasis of recti abdominis: an original technique of prosthesis repair of the abdominal wall [in Italian]. G Chir. 2007;28(5):187-198.

4. Heniford BT, Park A, Ramshaw BJ, Voeller G. Laparoscopic repair of ventral hernias: nine years' experience with 850 consecutive hernias. Ann Surg. 2003;238(3):391-400.

5. Sharma A, Mehrotra M, Khullar R, Soni V, Baijal M, Chowbey PK. Laparoscopic ventral/incisional hernia repair: a single centre experience of 1,242 patients over a period of 13 years. Hernia. 2011;15(2):131-139.

6. Karthikesalingam A, Kitcat M, Malata CM. Abdominoplasty in patients with and without pre-existing scars: a retrospective comparison. J Plast Reconstr Aesthet Surg. 2011; 64(3):369-374.

7. Tadiparthi S, Shokrollahi K, Doyle GS, Fahmy FS. Rectus sheath plication in abdominoplasty: assessment of its longevity and a review of the literature. J Plast Reconstr Aesthet Surg. 2012;65(3):328-332.

8. Blatnik JA, Harth KC, Aeder MI, Rosen MJ. Thirty-day readmission after ventral hernia repair: predictable or preventable? Surg Endosc. 2011;25(5):1446-1451.

9. Palanivelu C, Rangarajan M, Jategaonkar PA, Amar V, Gokul KS, Srikanth B. Laparoscopic repair of diastasis of recti using the "Venetian blinds" technique of plication with prosthetic reinforcement: a retrospective study. Hernia. 2009; 13(3):287-292.

10. Franklin ME Jr, Gonzalez JJ Jr, Glass JL, Manjarrez A. Laparoscopic ventral and incisional hernia repair: an 11-year experience. Hernia. 2004;8(1):23-27.
11. Tran HM. Safety and efficacy of single incision laparoscopic surgery for total extraperitoneal inguinal hernia repair. JSLS. 2011;15(1):47-52.

12. Tran HM. Robotic single port hernia surgery. JSLS. 2011; 15(3):309-314.

13. Tran HM. Demonstrated safety and efficacy of laparoendoscopic single site surgery for abdominal wall hernias. JSLS. 2012; 16(2):242-249.

14. Bozola AR, Psillakis JM. Abdominoplasty: a new concept and classification for treatment. Plast Reconstr Surg. 1988;82(6): 983-993.

15. Tran HM, Saliba L, Chandraratnam E, Turingan I, Hawthorne W. Strategies to minimize adhesions to intraperitoneally placed mesh in laparoscopic ventral hernia repair. JSLS. 2012;16(1):89-94.

16. Ahmed I, Ciancio F, Ferrara V, et al. Current status of singleincision laparoscopic surgery: European experts' views. Surg Laparosc Endosc Percutan Tech. 2012;22(3):194-199.

17. Sajid MS, Ladwa N, Kalra L, Hutson KK, Singh KK, Sayegh M. Single-incision laparoscopic cholecystectomy versus conventional laparoscopic cholecystectomy: meta-analysis and systematic review of randomized controlled trials. World J Surg. 2012; 36(11):2644-2653.

18. Grønvold LB, Spasojevic M, Nesgaard JM, Ignjatovic D. Single-incision laparoscopic versus conventional laparoscopic ventral hernia repair: a comparison of short-term surgical results. Surg Laparosc Endosc Percutan Tech. 2012;22(4):354-357.

19. Rehman H, Mathews T, Ahmed I. A review of minimally invasive single-port/incision laparoscopic appendectomy. $J$ Laparoendosc Adv Surg Tech A. 2012;22(7):641-646.

20. Li P, Wang DR, Wang LH, Li YK, Chen J. Single-incision laparoscopic surgery vs. multiport laparoscopic surgery for COlectomy: a meta-analysis of eleven recent studies. Hepatogastroenterology. 2012;59(117):1345-1349.

21. Kaouk JH, Autorino R, Kim FJ, et al. Laparoendoscopic single-site surgery in urology: worldwide multi-institutional analysis of 1076 cases. Eur Urol. 2011;60(5):998-1005.

22. Koyanagi T, Motomura S. Single-incision laparoscopically assisted vaginal hysterectomy: operative outcomes and its learning curve. Exp Ther Med. 2011;2(5):867-871. 